UCRL-JC-122439

PREPRINT

$$
\text { CONF-9510223--3-Vugraphis }
$$

\title{
Turbulent Mix Experiments
}

Guy Dimonte

TLEERVED PEB 201998

(). S 5

This paper was prepared for submittal to the 10th Biennial Nuclear Explosives Design Physics Conference (NEDPC)

Los Alamos, NM

October 30 - November 3, 1995

October 1995

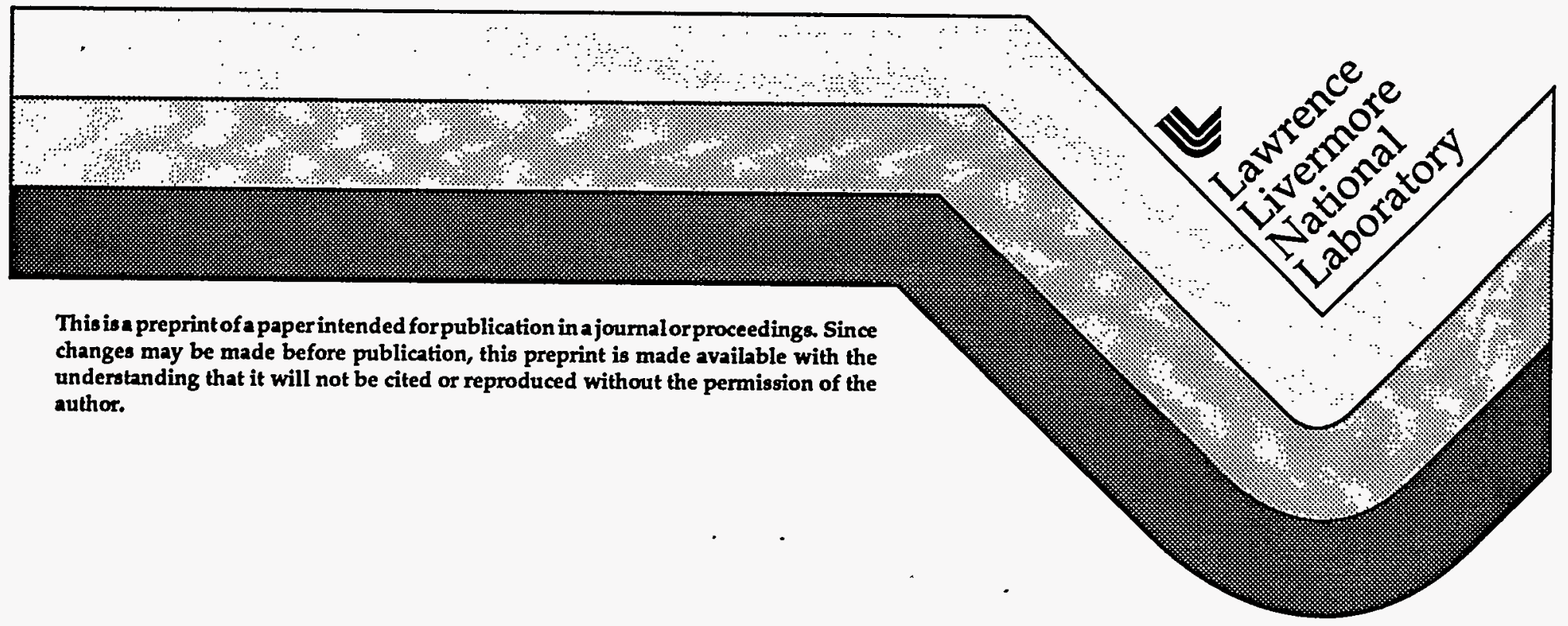




\section{DISCLAIMER}

This document was prepared as an account of work sponsored by an agency of the United States Government. Neither the United States Government nor the University of California nor any of their employees, makes any warranty, express or implied, or assumes anylegal liability or responsibility for the accuracy, completeness, orusefulness of any information, apparatus, product, or process disclosed, or represents that its use would not infringeprivately owned rights. Reference herein to any specificcommercial products, process, or service by trade name, trademark, manufacturer, or otherwise, does not necessarily constitute or imply its endorsement, recommendation, or favoring by the United States Government or the University of California. The views and opinions of authors expressed herein do not necessarily state or reflect those of the United States Government or the University of California', and shall not be used for advertising or product endorsement purposes. 


\author{
Guy Dimonte \\ Marilyn Schneider \\ Eric Frerking
}

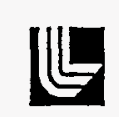

Lawrence Livermore National Laboratory

Livermore, CA 94551

Don Nelson, Sam Weaver, Judy Ticehurst, Carl Allison, Jim Morrison, Scott Hulsey,

Allan Susoeff, Ron Hawke, Bruce Remington, Jan Batteaux

MX_01

Objective of mix program is to quantify turbulent mix scaling laws

- Rayleigh-Taylor instability

$$
h=\alpha A\left[\int \sqrt{g} d t\right]^{2}
$$

- Richtmyer-Meshkov instability

$$
h=\alpha_{\mathrm{PM}}(U \mathrm{t})^{\beta} \mathbf{h}_{\mathrm{o}}^{1-\beta}
$$

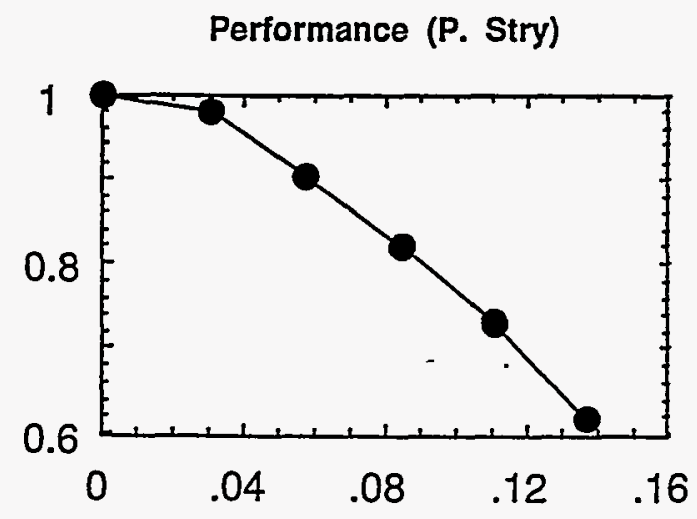

$\alpha$

Used to test mix models and simulation codes

Basic understanding required to scale to applications

This work was performed under the auspices of the U.S. Department of Energy by Lawrence Livermore National Laboratory under contract No. W-7405-Eng-48. 
- Strong radiatively driven shocks

Shaped Nova pulse

Foam tamper

- Planar geometry for radiography

Face-on \& side-on

- Seed perturbations at interface

Single mode

Multi-mode

Random

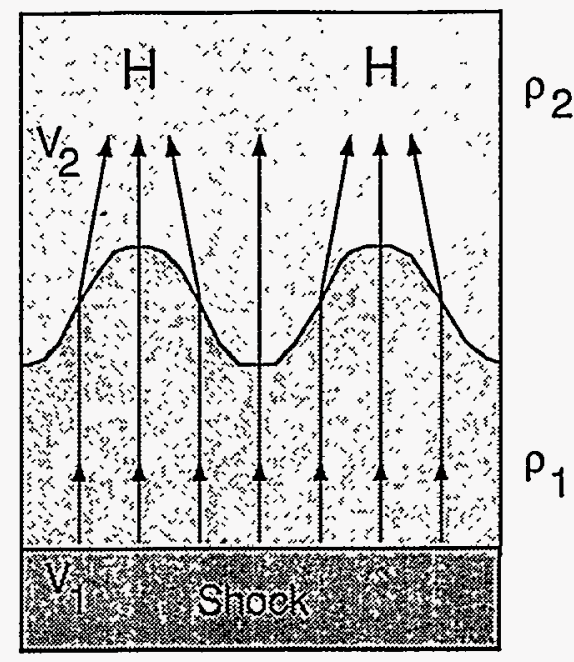

$$
A=\frac{\rho_{2}-\rho_{1}}{\rho_{2}+\rho_{1}}<0
$$

\section{Test linear theory full hydrodynamic simulations and models}

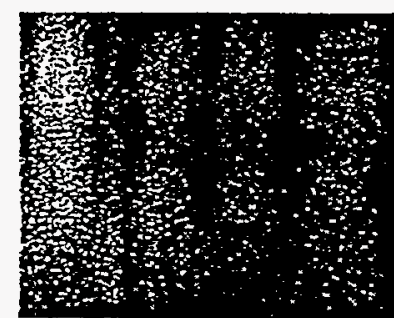

Face-on

Streaked

Radiograph

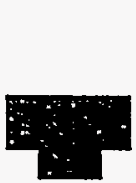

Side-on

2D Gated

Radiograph
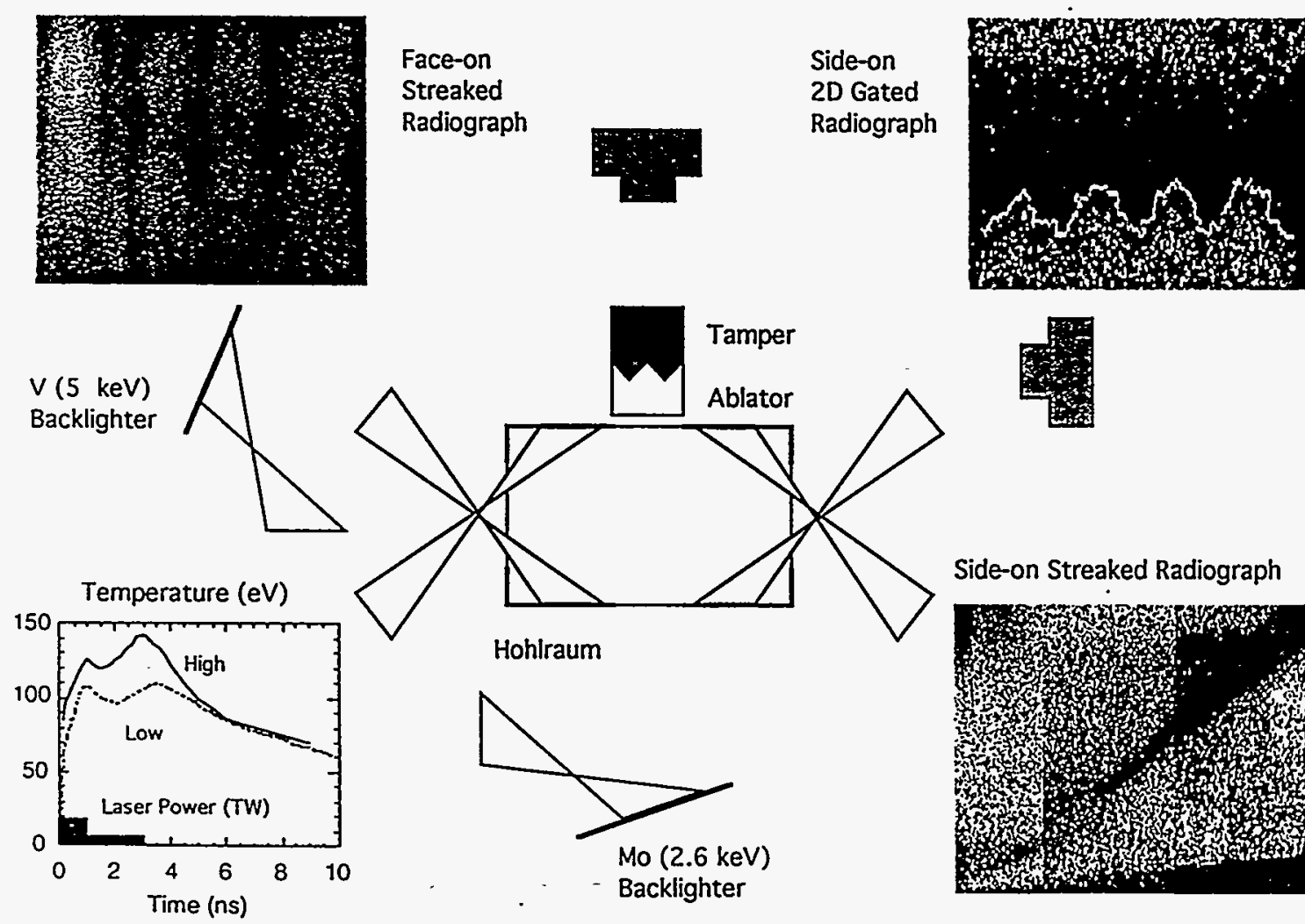

Hohlraum

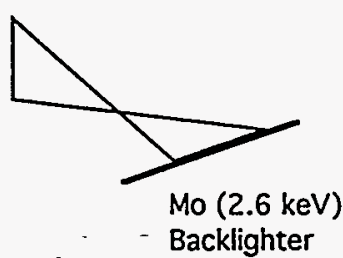

Backlighter
Tamper

Ablator

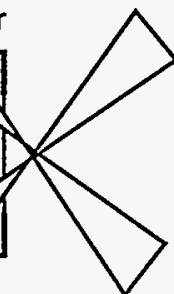

Side-on Streaked Radiograph

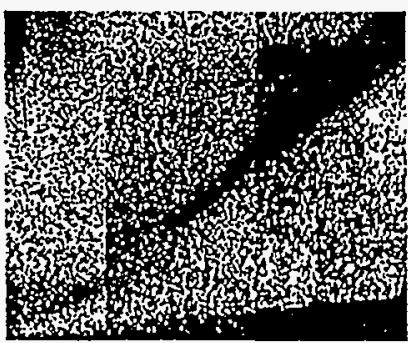

APS_04 
Agar tamper, high drive, $\lambda=100 \mu \mathrm{m}, \eta_{0}=10 \mu \mathrm{m}$

Fourier analysis of face-on \& side-on radiographs

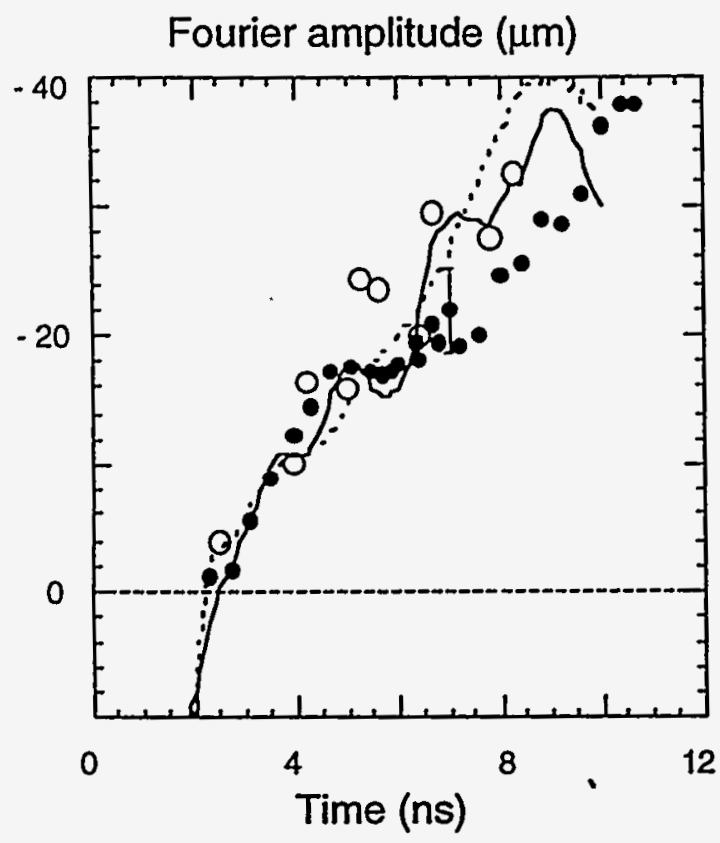

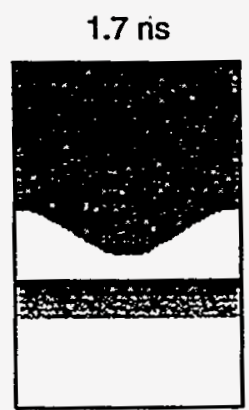

$4.5 \mathrm{~ns}$

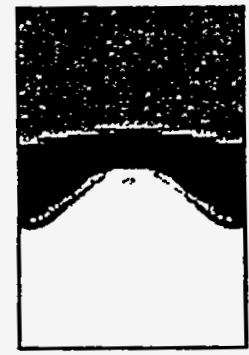

$2.2 \mathrm{~ns}$

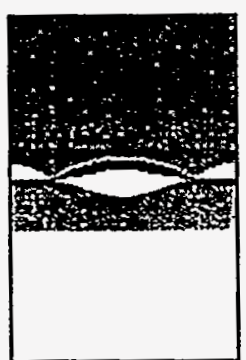

$7.0 \mathrm{~ns}$

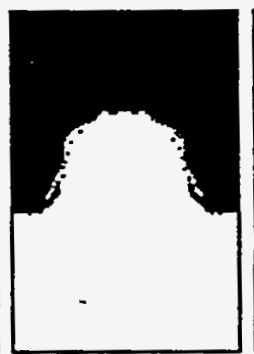

$3.0 \mathrm{~ns}$

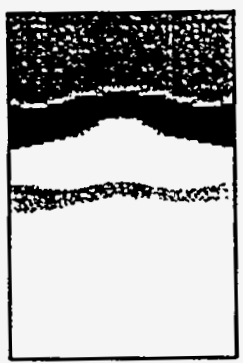

10 ns

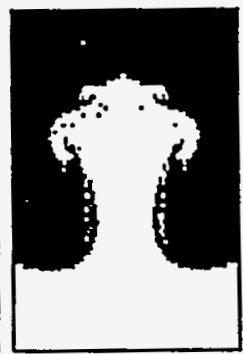

APS_06

Growth rates from Nova and CALE agree for a variety of conditions
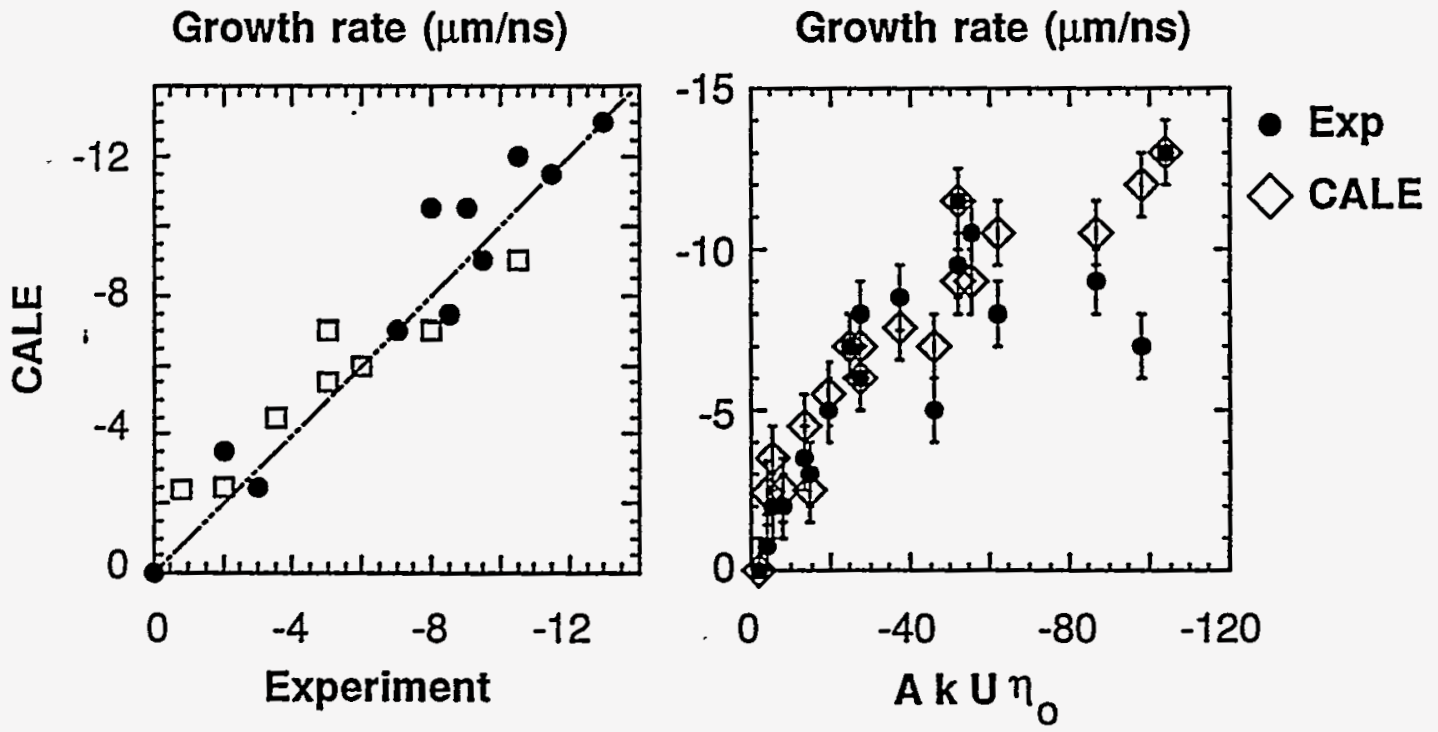

$\eta_{0}<0.15 \lambda$, growth rate proportional to $A K \cup \eta_{0}$ (impulsive models)

$\eta_{0}>0.15 \lambda$, growth rate limited to $15 \mu \mathrm{m} / \mathrm{ns}\left(V_{2}-U\right)$ 
Experimental linear growth rates $\left(\eta_{0}<0.15 \lambda\right)$

agree with Meyer-Blewett model for $\mathrm{A}<0$

Growth rates $(\mu \mathrm{m} / \mathrm{ns})$

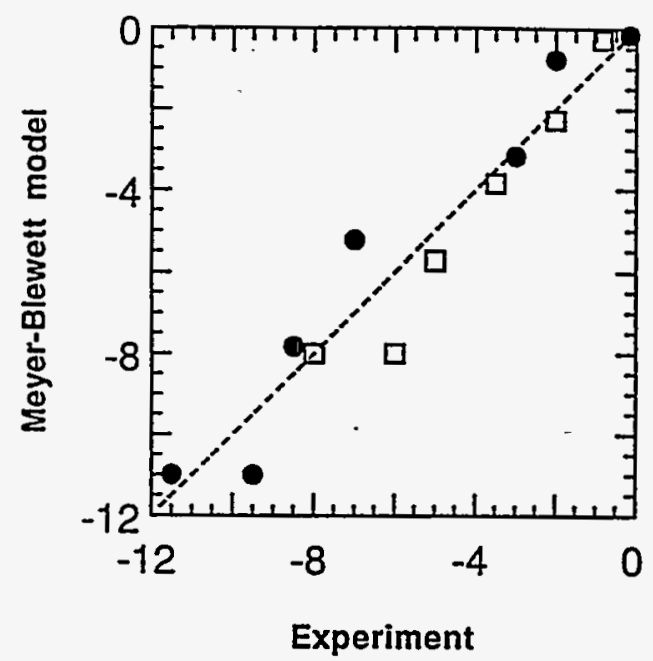

Meyer-Blewett model $(\mathrm{A}<0)$

$$
\frac{d \eta}{d t} \sim A^{*} k U \frac{\eta_{0}+\eta_{0}^{*}}{2}
$$

Richtmyer model $(\mathrm{A}>0)$

$$
\begin{aligned}
& \frac{d \eta}{d t} \sim A^{*} k U \eta_{0}^{*}>0 \\
& \eta_{0}^{*} / \eta_{0} \sim\left(1-U / V_{1}\right)<0
\end{aligned}
$$

HIGH COMPRESSION (Mach $>3$ ) required tor módels to obtain different sign of gi owith rate

Pertubation spike cannot overtake transmitted shock without causing significant drag \& nonlinearities

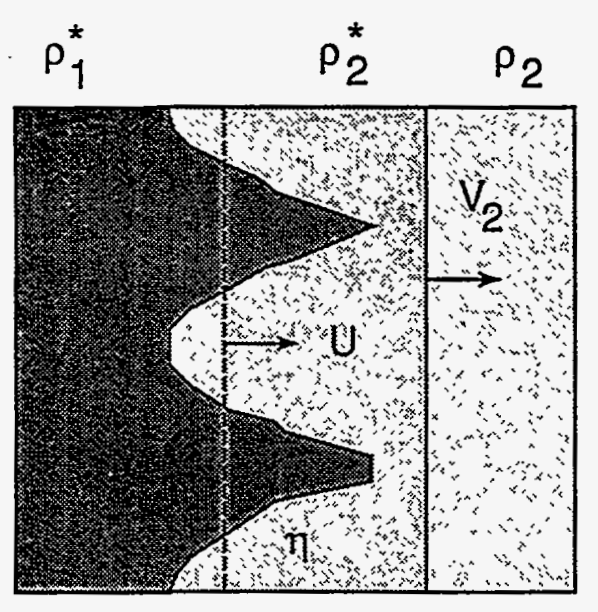

Interface Shock
For perturbation spike to remain behind transmitted shock

$$
\mathrm{U}+\mathrm{d} \eta / \mathrm{dt}<\mathrm{V}_{2}
$$

Growth rate limit at large amplitude

$$
A^{*} k U \eta_{\text {eff }}^{*}<V_{2}-U
$$

Affects experimental deșign on Nova

May be important for multiple shocks 

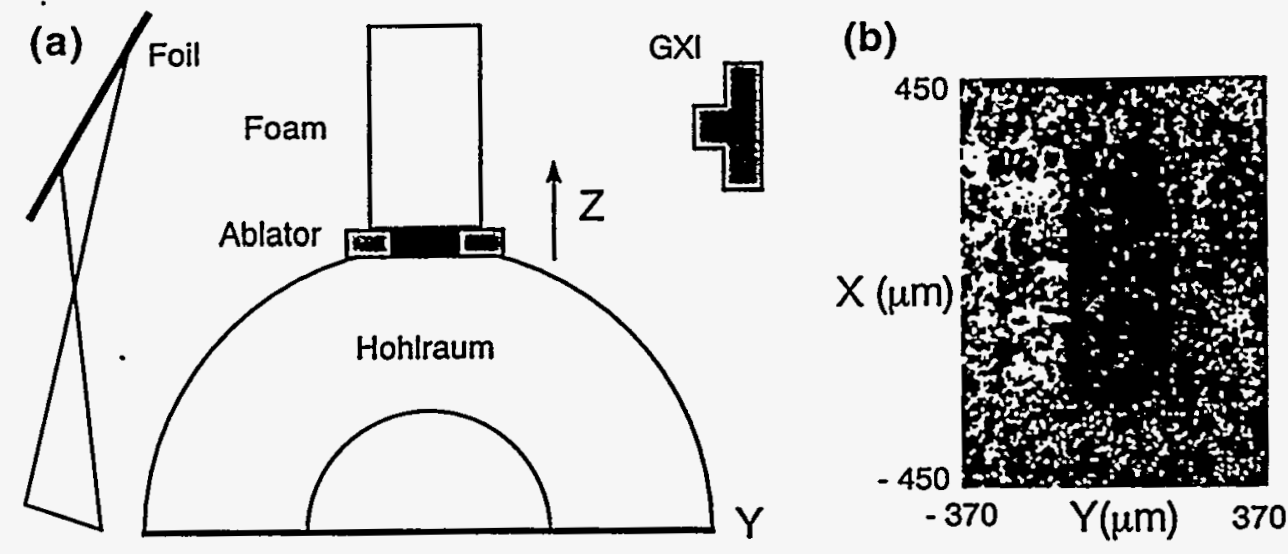

Figure 5: (a) Experimental configuration and (b) ablator

Figure 6: Side-on radiographs and $\mathrm{x}$-ray transmission profiles

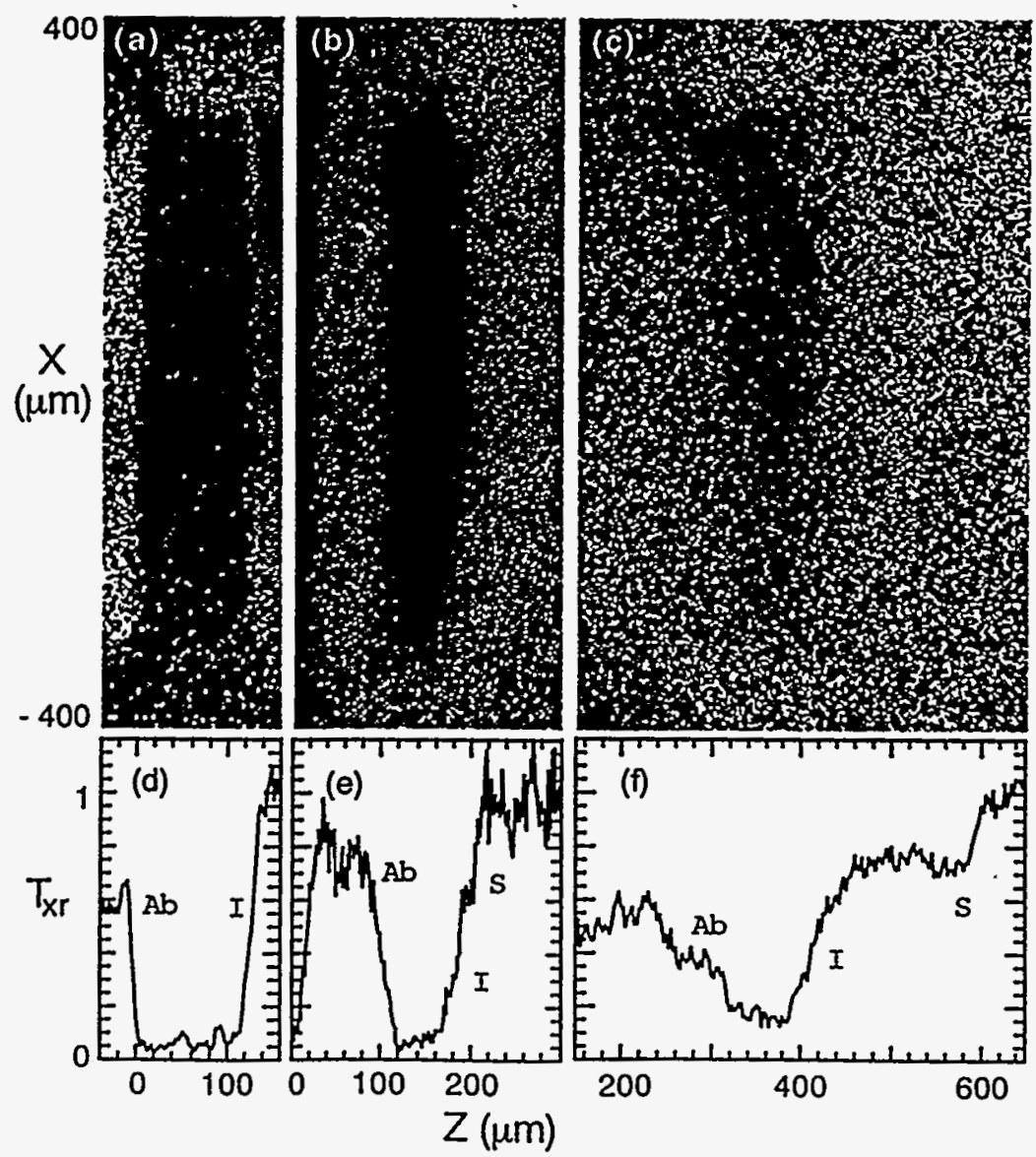


Figure 7: Side-on images and x-ray transmission profiles from 2D CALE simulation

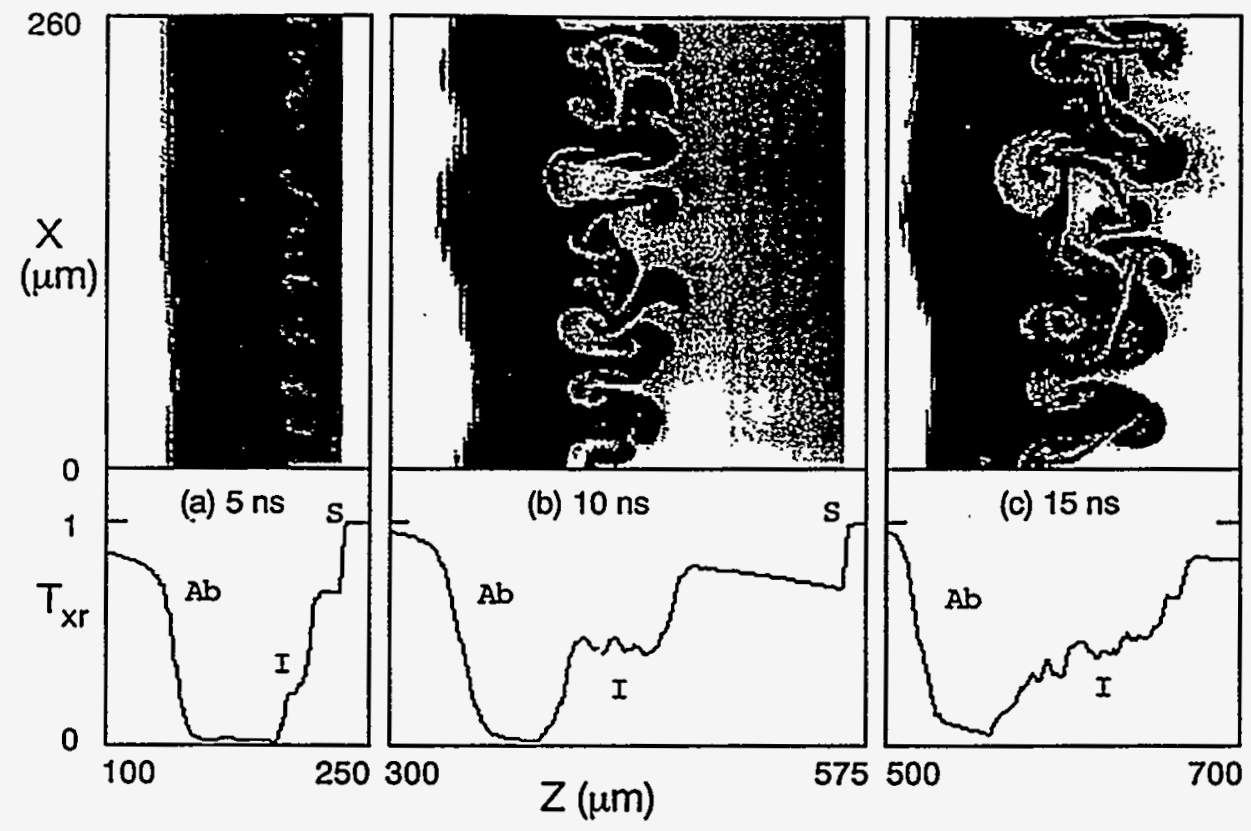

Turbulent mix width obeys power law scaling consistent with bubble merger or dynamic scale model

* Power law observed

$$
\begin{aligned}
& h \sim(U \delta t)^{\beta} \\
& \beta \sim 0.6 \pm 0.1
\end{aligned}
$$

- Dynamic scale model

$$
\begin{aligned}
& d h / d t \sim A k \cup h_{0} \\
& <k h>\sim \text { constant } \\
& h \sim\left(A h_{0} U \delta t\right)^{0.5}
\end{aligned}
$$

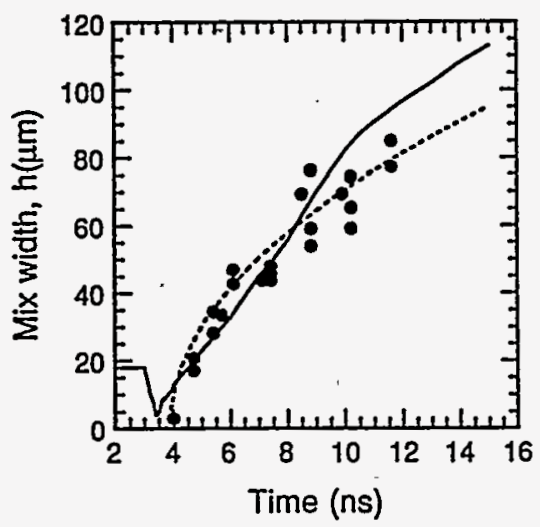

PRL 74, 4855 (1995) 
Test mix scaling laws

$$
\begin{aligned}
& h=\alpha A\left[\int \sqrt{g} d t\right]^{2} \\
& h=\alpha_{R M}(U t)^{\beta} h_{0}^{1-\beta}
\end{aligned}
$$

- Linear electric motor (LEM)

Variable acceleration

Turbulent $\left(L>D>\lambda_{\text {min }}\right.$ )

Incompressible

Optical diagnostics

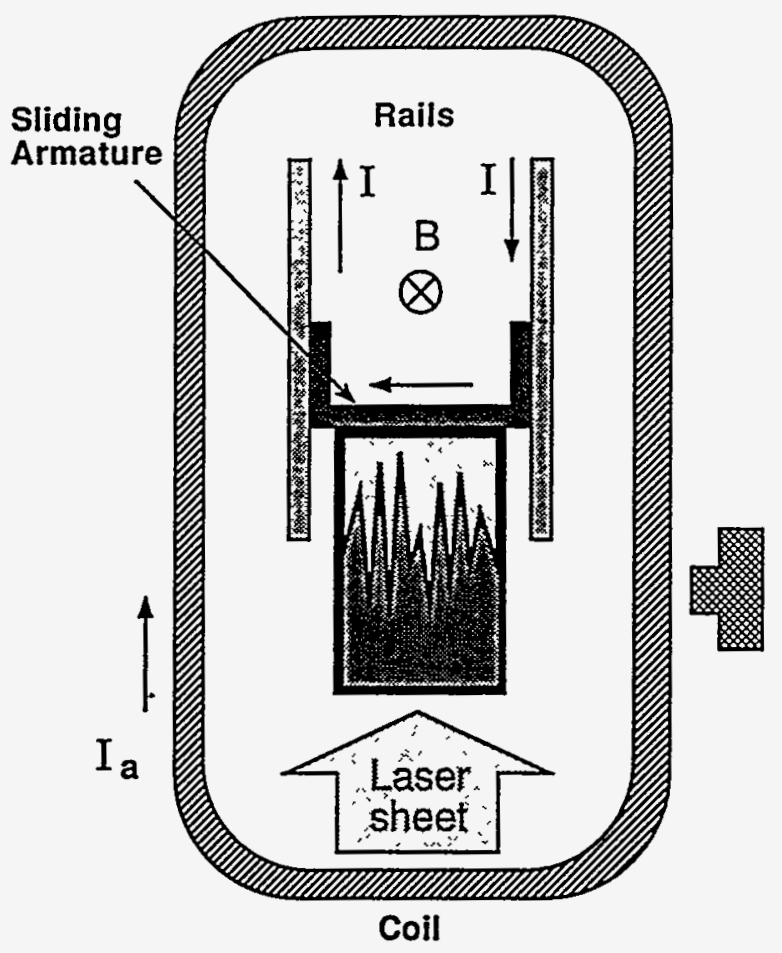

LEM_O2

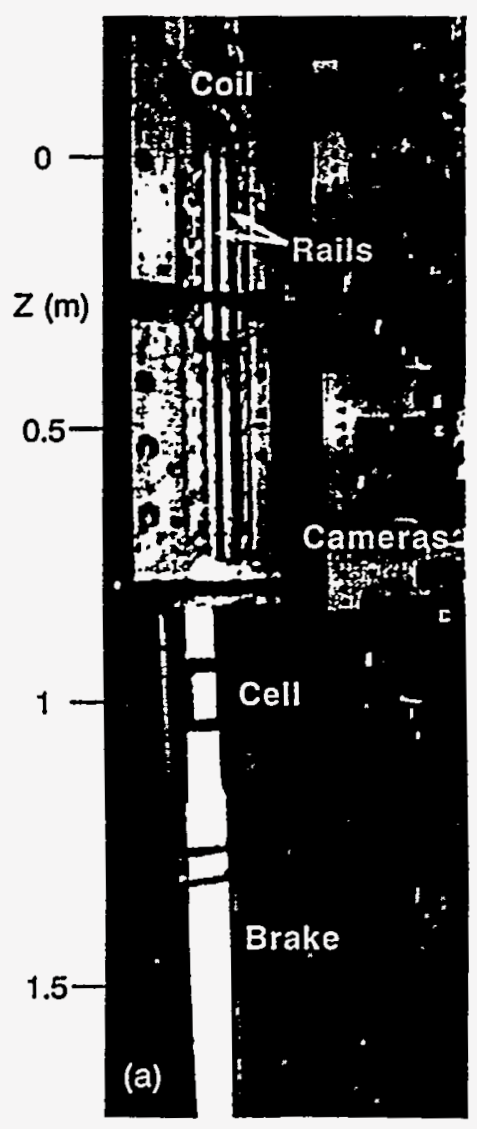

(b) Cell in accelerator

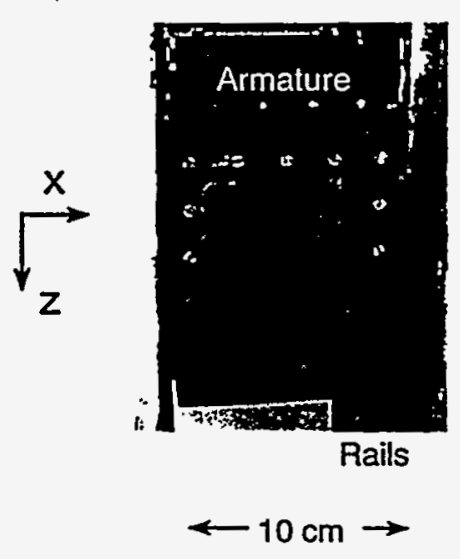

(c) Cell in brake

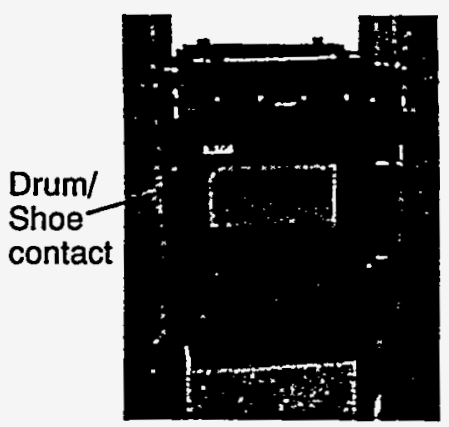

Figure 10: Axial view of LEM (a) and close-up view of cell $(b, c)$ 

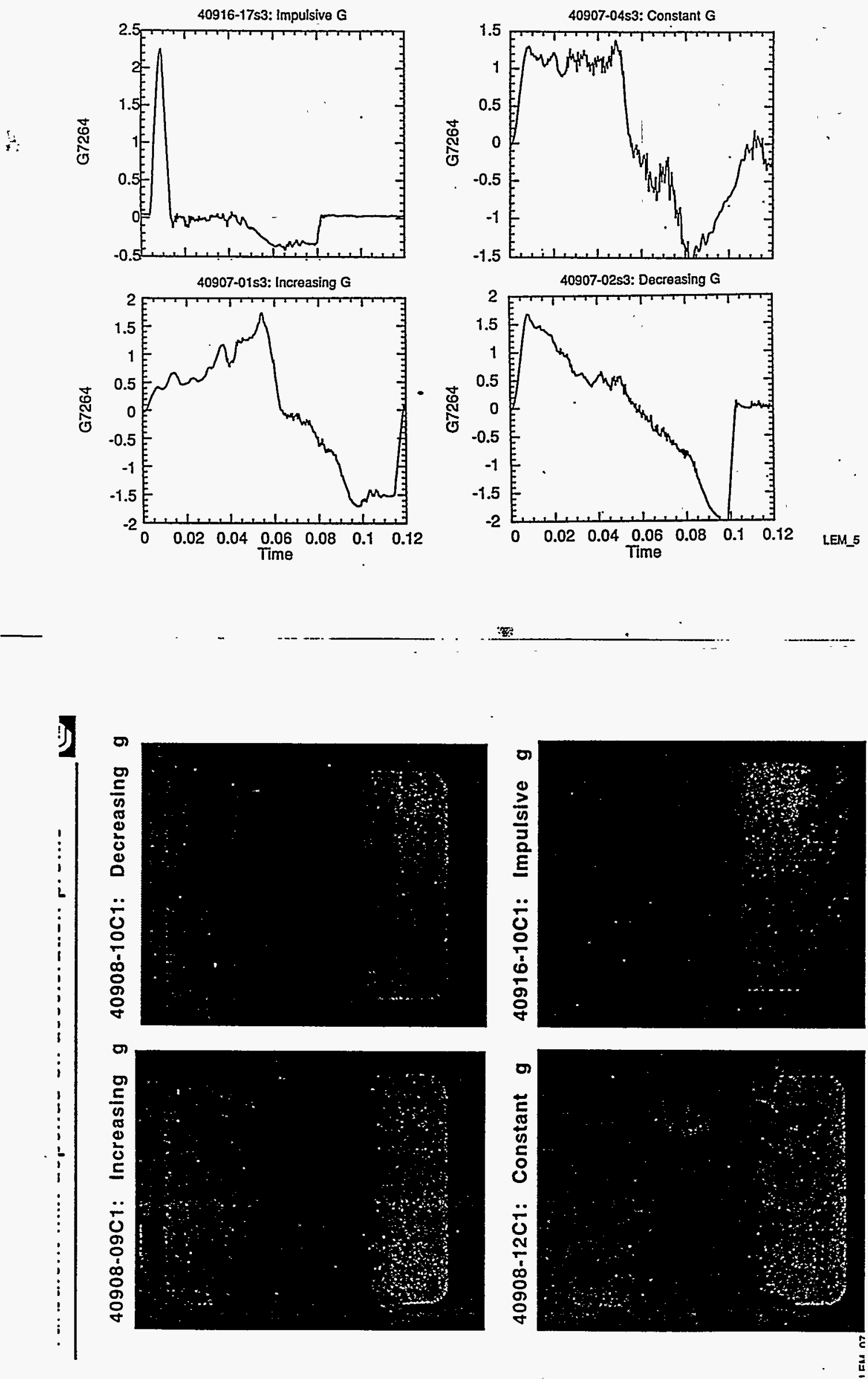
Bubble penetration proportional to displacement

$$
\begin{aligned}
& \mathbf{h}=\alpha \mathrm{Ag} \mathrm{t} \mathbf{t}^{2} \\
& \alpha \sim 0.06
\end{aligned}
$$

ALE simulations yield

$$
\alpha \sim 0.044
$$

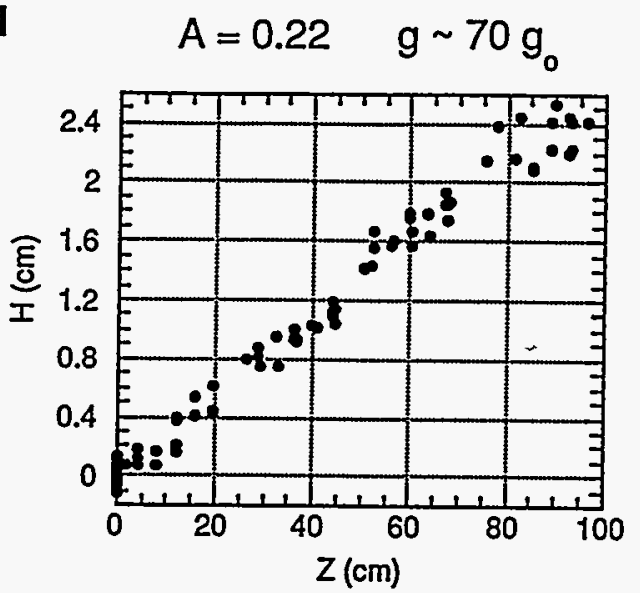

Bubble penetration with impulsive acceleration

$$
A=0.22 \quad g \sim 700 g_{0} \quad Z_{0} \sim 17 \mathrm{~cm}
$$

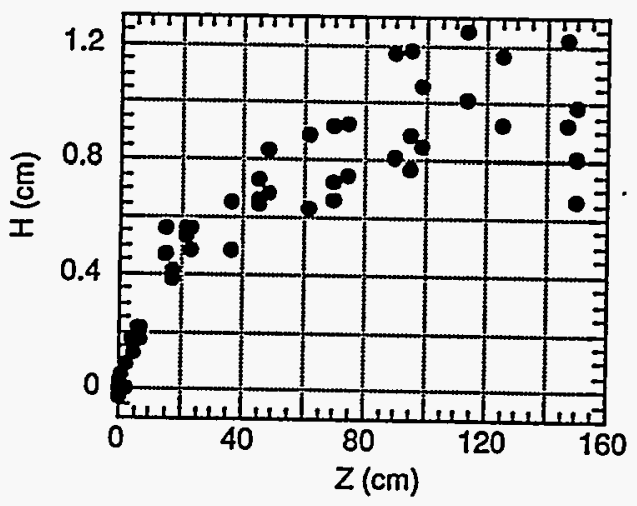

$$
\begin{aligned}
& h \sim h_{0}\left(Z / Z_{0}\right)^{\beta} \\
& h_{0} \sim 2 \alpha A Z_{0}
\end{aligned}
$$

$$
\alpha \sim 0.06 ; \quad, \beta \sim 0.5
$$



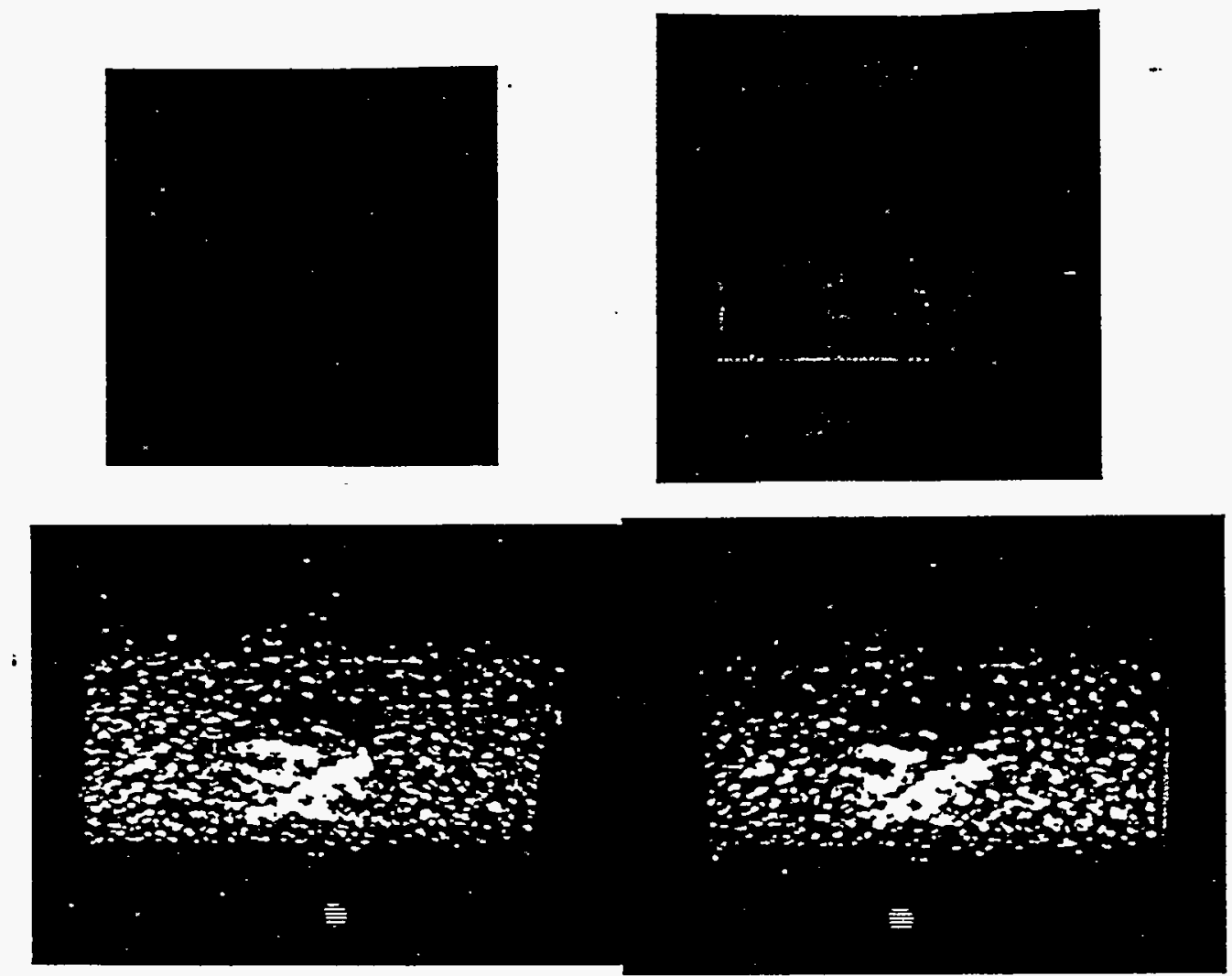

Mix experiments test models and simulations

- Single wavelength RM experiments (Nova) find 2 regimes

$$
\begin{gathered}
k \eta_{0}<1: \text { growth rate consistent with } \\
\text { Meyer-Blewett for } A<0
\end{gathered}
$$

$$
\frac{\mathrm{d} \eta}{\mathrm{dt}} \sim \mathrm{A}^{*} \mathrm{k}_{0} \frac{\eta_{0}+\eta_{0}^{*}}{2}
$$

$k \eta_{0}>1$ : growth rate approaches limiting value $\left(V_{2}-U\right)$

- RM experiments (Nova) with 3D random perturbations find

$$
h=\alpha(U) \hat{t} h_{0}^{\beta}-\beta
$$$$
\beta \sim 0.6 \pm 0.1
$$

- LEM for arbitrary acceleration profiles \& detailed diagnosis

$$
h \sim 0.06 A\left[\int \sqrt{g(t)} d t\right]^{2} \quad h \sim(U t)^{0.5}
$$


Technical Information Department . Lawrence Livermore National Laboratory University of California - Livermore, California 94551

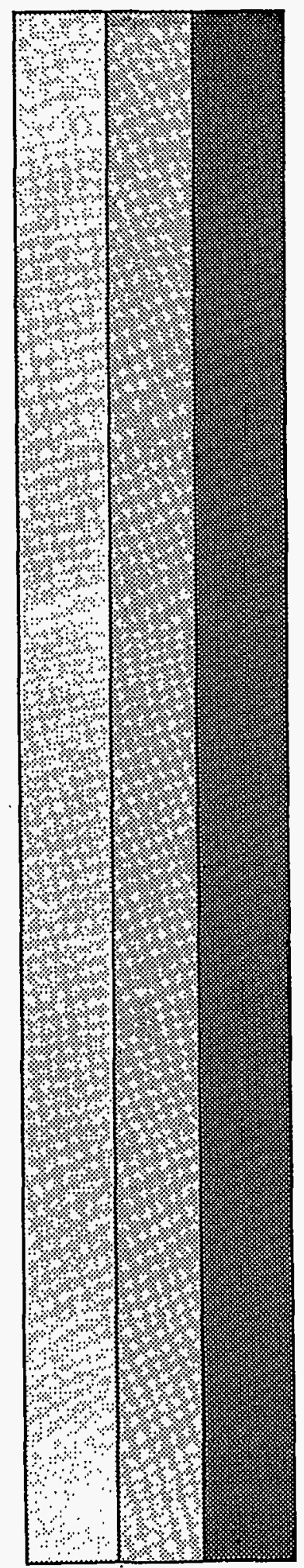

